\title{
Publisher Correction: A database of the coseismic effects following the 30 October 2016 Norcia earthquake in Central Italy
}

\author{
Fabio Villani ${ }^{1}$, Riccardo Civico ${ }^{1}$, Stefano Pucci ${ }^{1}$, Luca Pizzimenti ${ }^{1}$, Rosa Nappi ${ }^{1}$, \\ Paolo Marco De Martini ${ }^{1} \&$ the Open EMERGEO Working Group
}

Correction to: Scientific Data https://doi.org/10.1038/sdata.2018.49, published online 27 March 2018

In the original version of the Data Descriptor the surname of author Anne Socquet was misspelled. This has now been corrected in the HTML and PDF versions of the Data Descriptor.

Some authors were also not appropriately associated with their affiliations in the HTML version, due to formatting errors made by the publisher. This has now been corrected in the HTML version of the Data Descriptor, the affiliations in the PDF were correct from the time of publication.

(c) (i) Open Access This article is licensed under a Creative Commons Attribution 4.0 International License, which permits use, sharing, adaptation, distribution and reproduction in any medium or format, as long as you give appropriate credit to the original author(s) and the source, provide a link to the Creative Commons license, and indicate if changes were made. The images or other third party material in this article are included in the article's Creative Commons license, unless indicated otherwise in a credit line to the material. If material is not included in the article's Creative Commons license and your intended use is not permitted by statutory regulation or exceeds the permitted use, you will need to obtain permission directly from the copyright holder. To view a copy of this license, visit http://creativecommons.org/licenses/by/4.0/.

(C) The Author(s) 2019 


\section{the Open EMERGEO Working Group}

Fabio Villani ${ }^{1}$, Riccardo Civico ${ }^{1}$, Stefano Pucci ${ }^{1}$, Luca Pizzimenti ${ }^{1}$, Rosa Nappi ${ }^{1}$, Paolo Marco De Martini $^{1}$, F. Agosta ${ }^{2}$, G. Alessio ${ }^{1}$, L. Alfonsi ${ }^{1}$, M. Amanti ${ }^{3}$, S. Amoroso ${ }^{1}$, D. Aringoli ${ }^{4}$, E. Auciello ${ }^{5}$, R. Azzaro ${ }^{1}$, S. Baize ${ }^{6}$, S. Bello ${ }^{5}$, L. Benedetti ${ }^{7}$, A. Bertagnini ${ }^{1}$, G. Binda ${ }^{8}$, M. Bisson ${ }^{1}$, A. M. Blumetti ${ }^{3}$, L. Bonadeo ${ }^{8}$, P. Boncio ${ }^{5}$, P. Bornemann ${ }^{9}$, S. Branca ${ }^{1}$, T. Braun ${ }^{1}$, F. Brozzetti ${ }^{5}$, C. A. Brunori ${ }^{1}$, P. Burrato ${ }^{1}$, M. Caciagli ${ }^{1}$, C. Campobasso ${ }^{3}$, M. Carafa ${ }^{1}$, F. R. Cinti ${ }^{1}$, D. Cirillo ${ }^{5}$, V. Comerci ${ }^{3}$, L. Cucci ${ }^{1}$, R. De Ritis ${ }^{1}$, G. Deiana ${ }^{4}$, P. Del Carlo ${ }^{1}$, L. Del Rio ${ }^{10}$, A. Delorme ${ }^{11}$, P. Di Manna $^{3}$, D. Di Naccio ${ }^{1}$, L. Falconi ${ }^{12}$, E. Falcucci ${ }^{1}$, P. Farabollini ${ }^{4}$, J. P. Faure Walker ${ }^{13}$, F. Ferrarini $^{5}$, M. F. Ferrario ${ }^{9}$, M. Ferry ${ }^{14}$, N. Feuillet ${ }^{11}$, J. Fleury ${ }^{7}$, U. Fracassi ${ }^{1}$, C. Frigerio ${ }^{8}$, F. Galluzzo $^{3}$, R. Gambillara ${ }^{8}$, G. Gaudiosi ${ }^{1}$, H. Goodall ${ }^{15}$, S. Gori ${ }^{1}$, L. C. Gregory ${ }^{15}$, L. Guerrieri ${ }^{3}$, S. Hailemikael ${ }^{12}$, J. Hollingsworth ${ }^{16}$, F. lezzi ${ }^{17}$, C. Invernizzi ${ }^{4}$, D. Jablonská ${ }^{4}$, E. Jacques ${ }^{11}$, H. Jomard ${ }^{6}$, V. Kastelic ${ }^{1}$, Y. Klinger ${ }^{11}$, G. Lavecchia ${ }^{5}$, F. Leclerc ${ }^{18}$, F. Liberi ${ }^{5}$, A. Lisi ${ }^{1}$, F. Livio ${ }^{8}$, L. Lo Sardo ${ }^{19}$, J. P. Malet ${ }^{20}$, M. T. Mariucci ${ }^{1}$, M. Materazzi ${ }^{4}$, L. Maubant ${ }^{16}$, F. Mazzarini ${ }^{1}$, K. J. W. McCaffrey ${ }^{21}$, A. M. Michetti ${ }^{8}$, Z. K. Mildon ${ }^{13}$, P. Montone ${ }^{1}$, M. Moro ${ }^{1}$, R. Nave ${ }^{1}$, M. Odin ${ }^{20}$, B. Pace ${ }^{22}$, S. Paggi ${ }^{4}$, N. Pagliuca ${ }^{1}$, G. Pambianchi ${ }^{4}$, D. Pantosti ${ }^{1}$, A. Patera ${ }^{1}$, E. Pérouse ${ }^{7}$, G. Pezzo ${ }^{1}$, L. Piccardi² ${ }^{23}$, P. P. Pierantoni ${ }^{4}$, M. Pignone ${ }^{1}$, S. Pinzi ${ }^{1}$, E. Pistolesi ${ }^{4}$, J. Point ${ }^{20}$, L. Pousse $^{16}$, A. Pozzi ${ }^{8}$, M. Proposito ${ }^{12}$, C. Puglisi' ${ }^{12}$, I. Puliti ${ }^{24}$, T. Ricci ${ }^{1}$, L. Ripamonti ${ }^{25}$, M. Rizza7, G. P. Roberts ${ }^{17}$, M. Roncoroni ${ }^{25}$, V. Sapia ${ }^{1}$, M. Saroli ${ }^{1,19}{ }^{12}$ A. Sciarra ${ }^{1}$, O. Scotti ${ }^{6}$, G. Skupinski ${ }^{9}$, A. Smedile ${ }^{1}$, Anne Socquet ${ }^{16}$, G. Tarabusi ${ }^{1}$, S. Tarquini ${ }^{1}$, S. Terrana ${ }^{8}$, J. Tesson ${ }^{7}$, E. Tondi ${ }^{4}$, A. Valentini ${ }^{22}$, R. Vallone ${ }^{1}$, J. Van der Woerd ${ }^{20}$, P. Vannoli ${ }^{1}$, A. Venuti ${ }^{1}$, E. Vittori ${ }^{3}$, T. Volatili ${ }^{4}$, L. N. J. Wedmore ${ }^{13,15}$, M. Wilkinson ${ }^{26} \&$ M. Zambrano ${ }^{4}$

${ }^{2}$ Università della Basilicata, Potenza, 85100, Italy. ${ }^{3}$ Istituto Superiore per la Prevenzione e la Ricerca Ambientale (ISPRA), Roma, 00144, Italy. ${ }^{4}$ Università degli Studi di Camerino, Camerino, 62032, Italy. ${ }^{5}$ Università degli Studi "Gabriele D'Annunzio" di Chieti-Pescara, Centro Interuniversitario per l'Analisi Sismotettonica, Chieti, 66100, Italy. ${ }^{6}$ Institut de Radioprotection et Sûreté Nucléaire, BERSSIN, 92262, Fontenay-aux-Roses, France. ${ }^{7}$ Aix-Marseille Université, CEREGE CNRS-IRD UMR 34, 13545, Aix en Provence, France. ${ }^{8}$ Università degli Studi dell'Insubria, Como, 22100, Italy. ${ }^{9}$ Université de Strasbourg, CNRS, Lab Image Ville Environnement UMR, 7362, Strasbourg, France. ${ }^{10}$ Università degli Studi di Roma "La Sapienza", Roma, 00185, Italy. ${ }^{11}$ Institut de Physique du Globe de Paris, Sorbonne Paris Cité, Paris, 75005, France. ${ }^{12}$ Agenzia nazionale per le nuove tecnologie, l'energia e lo sviluppo economico sostenibile, (ENEA), Roma, 00196, Italy. ${ }^{13}$ Institute for Risk and Disaster Reduction, University College London, London, WC1E 6BT, UK. ${ }^{14}$ Géosciences Montpellier, Université de Montpellier, Montpellier, CNRS-UMR 5243 , France. ${ }^{15}$ University of Leeds, Leeds, LS2 9JS, UK. ${ }^{16}$ Université Grenoble Alpes, Université Savoie Mont Blanc, CNRS, IRD, IFSTTAR, ISTerre, Saint-Martin-d'Hères, 38400, Grenoble, France. ${ }^{17}$ Birkbeck University of London, London, WC1E 7HX, UK. ${ }^{18}$ Université Côte d'Azur, CNRS, Observatoire de la Côte d'Azur, IRD, Géoazur, Valbonne, 06560, France. ${ }^{19}$ Università degli Studi di Cassino e del Lazio Meridionale, DICeM, Cassino, 03043, Italy. ${ }^{20}$ Université de Strasbourg, CNRS, Institut de Physique du Globe de Strasbourg UMR, 7516, Strasbourg, France. ${ }^{21}$ Durham University, Durham, DH1, UK. ${ }^{22}$ Università degli Studi di Chieti-Pescara, DiSPUTer Chieti, Chieti, 66100, Italy. ${ }^{23}$ Consiglio Nazionale delle Ricerche, Istituto di Geoscienze e Georisorse (IGG), 50121, Firenze, Italy. ${ }^{24}$ Università degli Studi di Perugia, Perugia, 06123, Italy. ${ }^{25}$ SOGIN, Roma, 00185, Italy. ${ }^{26}$ Geospatial Research Ltd, Durham, DH1 4EL, UK. 